\title{
The Quality of Health Care and the Quality Of Life of Persons with Intellectual Disabilities: A Call for Disability-Inclusive Answers during Coronavirus Pandemics
}

\begin{abstract}
Santos $S^{*}$
UIDEF - Instituto da Educação, Faculdade de Motricidade Humana, Universidade de Lisboa, Portugal
\end{abstract}

*Corresponding author: Sofia Santos, UIDEF - Instituto da Educação, Faculdade de Motricidade Humana, Universidade de Lisboa, Portugal, Email: sofiasantos@fmh.ulisboa.pt

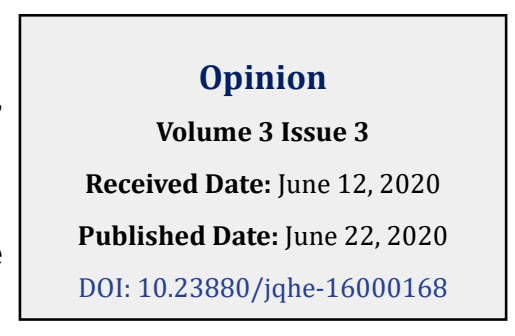

\section{Opinion}

The COVID-19 pandemic is a major public health issue that has changed the way persons are living. Many governments were initially focused on immediate solutions, especially at the health system level. However, all political and practical measures implementation should analyze the impact of coronavirus on life as a whole and not only in one life-domain. The current period does not affect everyone the same [1]. It revealed and intensified social inequalities and exclusion mechanisms. The debate should be based on evidences but should not be restricted to healthcare. It should involve economic interests as well as the rights of all citizens, including vulnerable population subgroups. Persons with disabilities are particularly disadvantaged by socioeconomics consequences of COVID-19 responses [2]. The purpose of this text is to reflect about the quality of health-social care measures and its potential impact in the quality of life (QOL) and personal outcomes of persons with intellectual disabilities (ID), during this period, focused on some thoughts that should not be forgot during the current coronavirus pandemic.

Besides the greater risk of infection from COVID-19, due to higher prevalence of comorbid health problems and personal habits, persons with ID are still confronted with several barriers (e.g.: attitudes, communication limitations) for social participation, and are at risk of seeing their personal outcomes and QOL been affected by immediate decisions. Persons with ID tend to depend on support of others for their daily life activities and within this pandemic period may find they more isolated, and without the main supports. The stayhome restrictions may create new risks for these persons' autonomy, health and participation/decision making. Those who are living in institutions may be even more vulnerable due to fast and easy spread of the disease.

The QOL of persons with ID is an emergent topic that has known an increased interest all over the world in policy, practice and research. QOL is a multidimensional construct composed by eight core domains organized in three main factors [3]: independence (personal development and selfdetermination domains), social participation (interpersonal relations, social inclusion and rights domains) and (physical, material and emotional) wellbeing. The significance and value of each domain may vary individually and so, the alignment between Rights and Quality of Life arouses, more than ever, the relevance of an active and meaningful participation by persons with ID towards measures' planning and implementation [4]. Also during emergency times. A number of articles are of particular relevance for the current situation: right to equality and non-discrimination (article $5^{\circ}$ ), right to safety (article 11. ${ }^{\circ}$ ), and right to the highest attainable standards of health (article 25. $\mathrm{o}$ ). But this opinion article will be focused on the right to freedom of expression and opinion, and access to information to full participation (article 21.. ).

Over recent years, efforts have been made to improve policies, diminish stigma and prejudice, aiming more intervention-evidences based and improve life conditions of persons with ID, based on ecological and holistic approaches, supports paradigm, and context framework. However, this emergency period may put at risk all the achievements so far. It is possible to identify some steps to inform what people with ID should do to protect themselves and other, during the current emergency. Accessible instructions and guidelines formats were designed and available. Organizations 
took decisions: persons with ID returned home to their families or those, who do not have any family net, remain institutionalized. How these organizations are raising awareness among persons with ID and their families for a more proper address to these subgroup needs? Persons with ID are just sent home to wait for things to get better? Or they are been given opportunity to maximum choices and control of their life's, during this period?

Some countries are good examples in prevention and well anticipated control actions for all, but what about the particular cases of persons who need more supports to make their own decisions. Measures cannot be judged to be good quality, just because they create safety [5], when its effectiveness or user's experiences are ignored [6]. Although the ascending phase, and while scientist are still discovering transmission modes and vaccines, we need to, continuously, think about the participation of persons with ID in all socioeconomic stages of contention process that affect their life. Persons with disabilities have the right to participate fully and effectively in decisions.

Nevertheless, solutions that are being provided may sometimes exclude own people voices, making these subgroup even more vulnerable and invisible than before. The rights of persons with ID are being considered or are still at risk? Independence, social inclusion and participation are being fully taking into account during this pandemic period? Students with ID are facing (more) barriers towards distance learning solutions (e.g., absence of equipment, lack of access to internet), which limits their opportunity to follow online school programs, restricts social interaction with peers and physical activity engagement, among other. Other concerns refer to COVID-10 impact on (un)employment, (un) sustainable social protection and support provision. How to overcome that? Were persons with intellectual disabilities included in crisis teams planning mitigation measures? Are persons with IDD contributing, somehow, to general decisions on supports provisions?

Research should analyses the impact of COVID-19 on the health and personal outcomes of persons with ID. How many persons with ID did the disease infect? How were they treated? More research is needed to identify, analyses and monitors what measures were taken in different countries in supports provision to persons with ID and how these persons' opinion and ideas were considered in all political-practical decisions and implementation. How were reorganized the provision of supports, both at home and in institutional settings? Do services met the client's needs according to their expectations and desires, through the provision of value products? Were staff ready-to-go to intervene through digital platforms? Were persons with ID prepared to deal with technological supports? What needs to change in future actions and practices?

The spread of COVID-19 will create major challenges for social service provision, unless actions area taken to avoid the increase of discrimination against people with ID. Persons with ID are disproportionately impacted. Persons with ID have the right to participate fully and effectively in these decisions. Therefore, this is a call for more participated actions: government, organizations, persons with ID and their families should work as partners to develop and implement measures to promote disability-inclusive responses to the current crisis. A human rights-based approach and a multidimensional and ethical framework for decision-making is required to ensure that persons with ID are not left behind in this period and are placed at the center of the response, as active agents. "A combination of mainstream and disability-specific measures is necessary to ensure systematic inclusion of persons with disabilities" [2]. Scientific evidences alone cannot drive decision-making. It is needed a balance between rights, interests and values. Reasonableness and transparency. Inclusiveness and responsiveness. Accountability, creativity and innovative solutions. Inclusive resources allocation and social stability. Community based-solutions. Work on disability inclusion is emergent to be prepared for future pandemics. Let's think about the long-term consequences of COVID-19 and others potential future pandemics, in health and wellbeing of all, without discrimination.

\section{References}

1. Arruda D (2020) Coronavirus, Inequality and Indifference. Journal of Quality in Health Care \& Economics 3(3): 1-3.

2. United Nations (2020) Policy Brief: a disability-inclusive response to COVID-19. MAY 2020. United Nations.

3. Schalock R, Verdugo M (2002) Handbook on Quality of Life for Human Service Practitioners. Washington, DC: American Association on Mental Retardation.

4. Verdugo MA, Navas P, Gomez LE, Schalock RL (2012) The concept of quality of life and its role in enhancing human rights in the field of intellectual disability. Journal of Intellectual Disability Research 56(11): 1036-1045.

5. Baguma JC, Obeta M (2020) Managing Quality in Health and Social Care Services; an Exemplary Review of a Center in London. Journal of Quality in Health Care \& Economics 3(2): 1-13.

6. Martin V, Henderson E (2001) Managing in health and social care. London: The Open University. 